\title{
A model for the screen-printing of Newtonian fluids
}

\author{
G. S. White (whitegs@maths.ox.ac.uk), C. J. W. Breward \\ (breward@maths.ox.ac.uk) and P. D. Howell \\ (howell@maths.ox.ac.uk) \\ Mathematical Institute, University of Oxford, 24-29 St Giles', Oxford OX1 3LB, \\ $U K$. \\ R. J. S. Young (richard.young@gbr.dupont.com) \\ Du Pont (UK) Ltd, Bristol Business Park, Coldharbour Lane, Frenchay, Bristol \\ $B S 161 Q D, U K$
}

19 April 2005

\begin{abstract}
A preliminary investigation into aspects of the off-contact screen-printing process is presented. A mathematical model for the printing of a thin film of Newtonian fluid is proposed, in which the screen is modelled as a permeable membrane, and the entire region above and below the screen is flooded. By drawing upon widely used industrial circuit printing practices, the distinguished limit of greatest interest to this industry is identified. Numerical and asymptotic solutions of this distinguished limit are presented that reproduce many of the features observed in industrial screen-printing.
\end{abstract}

Keywords: screen-printing, lubrication theory, asymptotic expansions

\section{Introduction}

Since the 1960s, electronic hybrid circuits have been produced by screenprinting thick film pastes. As with the rest of the electronics industry, there is a strong drive to increase circuit density and reduce feature size. This trend towards miniaturisation requires increasingly sophisticated paste rheologies and printing techniques.

Industrial screen-printing has developed from traditional silk-screen printing, and the processes still have much in common. Screen-printing, with its roots in the arts, has largely evolved by empirical evaluation without the benefit of conceptual and mathematical models. This experimental evaluation strategy is typified by the work of Kobs and Voigt [1], who listed over 50 variables, selected several of the most important ones and evaluated the effects of over 280 different parameter combinations in comparative printing tests. Every thick film print-run since has repeated the same basic testing strategy, although normally on a smaller scale. While this has allowed a huge empirical database to be compiled, universal rules for screen-printing have not been formulated because generalisation is difficult without theoretical models.

(C) 2005 Kluwer Academic Publishers. Printed in the Netherlands. 
The historical reliance upon empirical evaluation is made all the more surprising, since even a modest improvement in screen-printing technology would have a major impact on many technologies reliant upon micro-electronics.

One of the principal applications for screen printing today is to produce the current collection wires on the top of solar cells. The efficiency of such cells is directly related to the width of the collection wires, since their shadow prevents light reaching the solar cell. If their width could be reduced from $150 \mu \mathrm{m}$ to $75 \mu \mathrm{m}$ then, without any change in the base solar cell technology, the power output could be enhanced by as much as $1.5-2.0 \%$.

The development of models, albeit simple in the first instance, will lead to a finer understanding of key design elements of both the paste and the screen-printing process to enable the industry to fulfil its goals.

\section{Description of the industrial process}

Electronic hybrid circuits normally contain regions of conducting, insulating and dielectric materials. For the micro-electronics industry, screen-printing offers the most cost-effective means of patterning these diverse circuit materials.

The industrial screen-printing process uses a paste as a vehicle to transport metal and/or inorganic powders onto the required circuit pattern on the substrate. The pattern is formed by forcing the paste through a permeable screen whose 'pores' have been selectively occluded by a durable polymer. The screen is typically made from an ultra-thin stainless steel wire cloth and held under high tension over a solid metal frame. After printing, the circuit is formed by firing the printed paste to evaporate off the volatiles, pyrolyse remaining organic materials, and sinter any metal particles together.

There are two main variations of the process. The first is off-contact screen-printing, where the screen is held under high tension above the substrate; the second is contact screen-printing, where the screen is placed on the substrate. We will consider the off-contact case in this paper as it has become the de facto standard printing method in the micro-electronics industry. The contact case is generally not preferred as, with this method of printing, it is frequently observed that lifting the screen off the substrate destroys much of the desired fine-scale pattern resolution.

In off-contact screen-printing, a pool of paste is laid on top of the screen in front of a rubber squeegee. The squeegee is moved forward, deforming the screen downwards until it makes contact with the sub- 


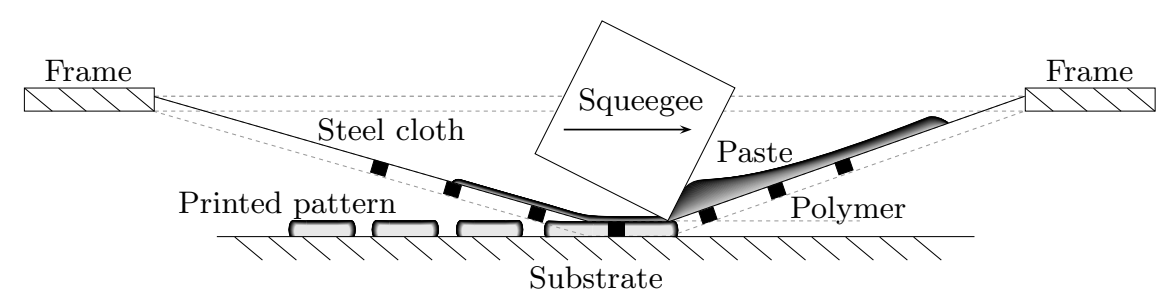

Figure 1. A sketch of the off-contact screen printing process. The screen has been labelled in terms of its two separate components, namely the steel cloth (sketched as a solid black line) and the emulsion (sketched as black boxes connected to the underside of the cloth). The arrow on the squeegee indicates that the squeegee would be travelling from left to right in the sketch. The unperturbed screen position is shown on the diagram as the straight dashed lines connecting either side of the frame.

strate. The paste is pushed along in front of the squeegee and through the screen pattern onto the substrate. The screen 'peels off' dynamically from the substrate at some distance behind the squeegee. The process is illustrated in Figure 1.

It is interesting to note that the printing practices of the microelectronics industry have developed in such a way as to minimise possible screen tension variations during the print. When fixing the wire cloth to the metal frame, the weave of the wire cloth is oriented to be at $45^{\circ}$ to the intended print direction. This ensures that the load is distributed over as many wires as possible at any given time. Further, it means that any steel wire supports the load for only a fraction of the total print stroke. This practice was originally adopted as it was found to increase the life-time of the screens.

The first attempts to gain a theoretical understanding of the process were made relatively recently in the 1980s. The most notable example of this early research is perhaps Riemer [2, 3, 4], who developed mathematical models of the screen-printing process based principally on the Taylor scraper model [5] for Newtonian fluids. These simple models were used to examine the effect of squeegee orientation upon the pressure in the paste ahead of the squeegee. Riemer's work has been well received by the screen-printing community and has been extended to include fluid leakage under the squeegee by Hunter [6], power-law fluids by Riedler [7] and Chapman et al. [8] and a gap between the scraper and the substrate by Jeong and Kim [9]. Despite their popularity, it is questionable what can be reliably inferred about screen-printing from these scraper models. They do not, for example, incorporate the permeability and flexibility of the screen, which are fundamental features of the process. Further, they cannot easily be 
extended to do so, since they rely upon finding solutions for Stokes flow by separation of variables - an approach only practicable in wedge geometries with simple boundary conditions.

In the light of these objections, Owczarek and Howland [10, 11] instead adopted a modelling approach based upon lubrication theory, more recently extended by Anderson et al. [12] to consider the printing of power-law fluids. None of these models, however, has taken into proper consideration the real geometry of the screen-printing process. Other attempts to gain insight into the printing process have relied upon numerical experiments, often focusing on easier (though related) printing/coating processes, such as stencil and contact screenprinting [13].

In this paper, we develop a model with a geometry that is similar to that of off-contact screen-printing. To ensure that this process remains our focus, we avoid the complications associated with non-Newtonian rheology and fluid/air interfaces by assuming that the fluid is Newtonian and that the regions above and below the screen are already flooded with paste. We also neglect the effects of the occluding polymer on the screen. Consequently, our model effectively concerns the second print of a Newtonian fluid during so-called 'double-wet' printing when the same substrate is printed over twice.

In Section 3, we describe the assumptions behind our model and derive the governing equations in non-dimensional form, identifying the dimensionless groups that determine the behaviour of the system. In Section 4, we derive a simplified model by taking a distinguished asymptotic limit that appears to be of most relevance to industrial screen-printing. We find that there are two regions to consider, and thus obtain an 'outer' problem describing the large-scale evolution of the screen and paste, and an 'inner' problem governing the behaviour near the squeegee tip. Numerical solutions to these problems are shown in Section 5, and their observed features are explained using asymptotic analysis in Sections 6 and 7. Finally, we draw our conclusions in Section 8.

\section{Problem formulation and scaling}

We consider a screen with undisturbed height $h_{f}$ above a planar substrate, as illustrated in Figure 2. The screen is held under tension $T$ from its ends a distance $L$ apart, and is deformed by a squeegee moving over the top with constant horizontal velocity $U$. Using Cartesian coordinates $(x, y)$, we denote the position of the lower surface of the squeegee by $y=H(x, t)$ and the position of the deformed screen by 


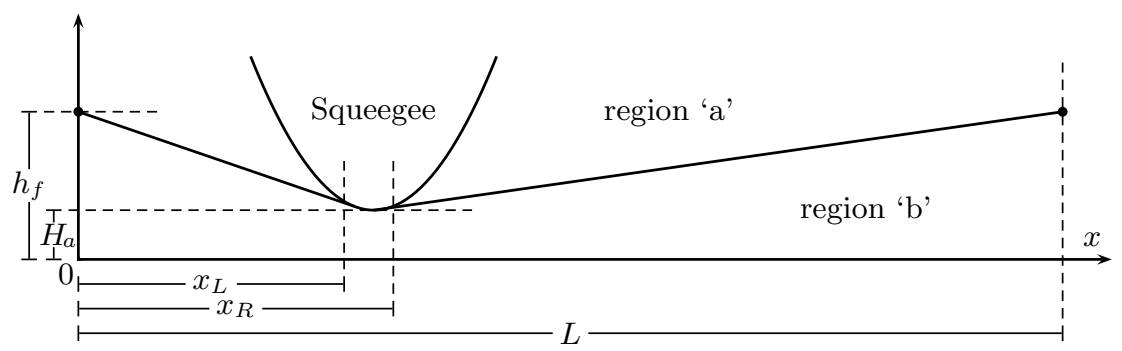

Figure 2. A sketch of the initial configuration of the model problem.

$y=h(x, t)$. The subscripts ' $a$ ' and ' $b$ ' are used to distinguish between the fluid pressures, $p$, and velocities, $\mathbf{v}=(u, v)$, above and below the screen respectively.

We assume that the screen has negligible bending stiffness and, in light of the discussion in Section 2 of industrial printing practices, that the screen tension $T$ remains constant throughout the print. Thus, where it is not in contact with the squeegee, a force balance across the screen gives

$$
p_{a}-p_{b}=T \kappa_{s c}
$$

where $\kappa_{s c}$ is the curvature of the screen.

We treat the screen as a semi-permeable membrane, transmitting a flux of paste that is proportional to the pressure difference across it. Hence the normal boundary conditions upon the paste velocity on either side of the screen are

$$
\begin{aligned}
& \left(\mathbf{v}_{a}-\mathbf{v}_{\text {screen }}\right) \cdot \mathbf{n}=-\Lambda\left(p_{a}-p_{b}\right), \\
& \left(\mathbf{v}_{b}-\mathbf{v}_{\text {screen }}\right) \cdot \mathbf{n}=-\Lambda\left(p_{a}-p_{b}\right),
\end{aligned}
$$

where $\mathbf{n}$ is the upward unit normal and $\Lambda$ is the screen permeability. We assume also that the tangential velocity is continuous across the screen, so that

$$
\begin{aligned}
& \left(\mathbf{v}_{a}-\mathbf{v}_{\text {screen }}\right) \cdot \mathbf{t}=0 \\
& \left(\mathbf{v}_{b}-\mathbf{v}_{\text {screen }}\right) \cdot \mathbf{t}=0
\end{aligned}
$$

where $\mathbf{t}$ is the unit tangent vector. This assumption is examined in more detail in Section 3.4 when we consider the operating parameter regime for industrial screen-printing.

From (2) and (3), we see that the normal and tangential velocity components are both continuous across the screen and, hence, that $\mathbf{v}_{a}$ and $\mathbf{v}_{b}$ are equal on the screen. The kinematic boundary condition for the screen height $h(x, t)$ may therefore be written as

$$
-h_{t}+\mathbf{v}_{a} \cdot\left(-h_{x}, 1\right)=-h_{t}+\mathbf{v}_{b} \cdot\left(-h_{x}, 1\right)=-\Lambda\left(p_{a}-p_{b}\right) .
$$


Note that the occurrence of ' $x$ ', ' $y$ ' or ' $t$ ' as a subscript signifies partial differentiation with respect to that variable. On all other surfaces, namely the substrate and squeegee, we assume that no-slip and no-penetration boundary conditions hold.

At the edge of the frame, we prescribe the screen height $h_{f}$ and set the flux of liquid below the screen to zero. Above the screen, we require that the pressure should be zero at the screen edge. Of course, where the squeegee and the screen come into contact, we require additional boundary conditions. At the a priori unknown points of contact, denoted by $x_{L}$ and $x_{R}$, we specify that the screen and its slope must be the same as those for the squeegee, that is, $h=H$ and $h_{x}=H_{x}$ at $x=x_{L}, x_{R}$.

\subsection{Further MODELLING ASSUMPTIONS}

We suppose that the aspect ratio $L / h_{f}$ is large and treat the paste as an incompressible Newtonian fluid with constant viscosity $\mu$ and negligible inertia. Under these assumptions, the fluid above and below the screen satisfies the lubrication equations

$$
\begin{aligned}
& p_{a x}=\mu u_{a y y}, \quad p_{a y}=0, \quad u_{a x}+v_{a y}=0, \\
& p_{b x}=\mu u_{b y y}, \quad p_{b y}=0, \quad u_{b x}+v_{b y}=0,
\end{aligned}
$$

The kinematic and dynamic boundary conditions for the fluid at the screen (1), (3) and (4) simplify to

$$
\begin{array}{ll}
-h_{t}+v_{a}=-\Lambda\left(p_{a}-p_{b}\right), & u_{a}=0, \quad p_{a}-p_{b}=T h_{x x}, \\
-h_{t}+v_{b}=-\Lambda\left(p_{a}-p_{b}\right), & u_{b}=0,
\end{array}
$$

on $y=h(x, t)$, while the no-slip and no-penetration conditions on the substrate and squeegee lead to

$$
\begin{array}{ll}
\left(u_{b}, v_{b}\right)=(0,0) & \text { on } y=0, \\
\left(u_{a}, v_{a}\right)=\left(U, H_{t}+U H_{x}\right) & \text { on } y=H(x, t) .
\end{array}
$$

The boundary conditions on the screen itself read

$$
\begin{array}{rll}
h(0, t)=h_{f}, & h\left(x_{L}, t\right)=H\left(x_{L}, t\right), & h_{x}\left(x_{L}, t\right)=H_{x}\left(x_{L}, t\right), \\
h(L, t)=h_{f}, & h\left(x_{R}, t\right)=H\left(x_{R}, t\right), & h_{x}\left(x_{R}, t\right)=H_{x}\left(x_{R}, t\right) .
\end{array}
$$

Using standard methods of lubrication theory [14, pages 65-66], we reduce the above system to a coupled set of Reynolds-type equations:

$$
(H-h)_{t}-\left[\frac{(H-h)^{3} p_{a x}}{12 \mu}-\frac{U(H-h)}{2}\right]_{x}+\Lambda\left(p_{a}-p_{b}\right)=0,
$$




$$
\begin{aligned}
h_{t}-\left[\frac{h^{3} p_{b x}}{12 \mu}\right]_{x}-\Lambda\left(p_{a}-p_{b}\right) & =0 \\
T h_{x x}-\left(p_{a}-p_{b}\right) & =0
\end{aligned}
$$

valid for $0 \leq x \leq L$.

It is worth noting that aspect ratio of the domain above the screen and away from the squeegee tip does not justify the use of lubrication theory. Instead we appeal to the fact that the pressure in this region is nearly constant and almost equal to the ambient pressure. It is also worth pointing out that (9)-(11) do not reduce to the familiar single-phase Reynolds equation if $T$ and $\Lambda$ are set to zero. This is a consequence of the boundary conditions (6) which constrain the tangential velocity to be zero on the screen.

\subsection{INITIAL CONDITIONS}

Initially, we assume that the system is at rest with contact between the squeegee and the screen and with both pressures equal to zero. Further, (11) implies that the screen is flat where not in contact with the squeegee. With this information, the condition of tangency at the contact points is enough to determine uniquely the initial configuration of the screen.

We suppose, for simplicity, that the squeegee is rigid, with a quadratic profile given by

$$
H=H_{a}+\frac{1}{2} \kappa_{s}\left(x-x_{i}-U t\right)^{2},
$$

where $\kappa_{s}, x_{i}$ and $H_{a}$ represent the curvature of, the initial position of, and height of the squeegee tip. This additional assumption enables us to express our initial condition for $h$ as

$$
h= \begin{cases}h_{f}-\kappa_{s} x\left(x_{i}-x_{L}\right) & 0<x<x_{L}, \\ H_{a}+\frac{1}{2} \kappa_{s}\left(x-x_{i}\right)^{2} & x_{L}<x<x_{R}, \\ h_{f}+\kappa_{s}(x-L)\left(x_{R}-x_{i}\right) & x_{R}<x<L,\end{cases}
$$

at $t=0$, where the left and right contact points, $x_{L}, x_{R}$, are given by

$$
\begin{aligned}
& x_{L}=\sqrt{x_{i}^{2}+2\left(H_{a}-h_{f}\right) / \kappa_{s}}, \\
& x_{R}=L-\sqrt{\left(L-x_{i}\right)^{2}+2\left(H_{a}-h_{f}\right) / \kappa_{s}} .
\end{aligned}
$$

The initial configuration for the screen and squeegee is illustrated in figure 2. 


\subsection{NON-DIMENSIONALISATION}

We non-dimensionalise with the following scalings

$$
\begin{aligned}
& x=L \bar{x}, \quad x_{i}=L \bar{x}_{i}, \quad x_{L, R}=L \bar{x}_{L, R}, \quad t=(L / U) \bar{t}, \\
& h=h_{f} \bar{h}, \quad H=\kappa_{s} L^{2} \bar{H}, \quad u_{a, b}=U \bar{u}_{a, b} \text {. }
\end{aligned}
$$

For consistency with the approximations that lead to (9), (10), we scale the pressures with

$$
p_{a}=\frac{\mu U}{\kappa_{s}^{2} L^{3}} \bar{p}_{a}, \quad p_{b}=\frac{\mu U L}{h_{f}^{2}} \bar{p}_{b} .
$$

The difference between the pressure scalings is a result of the different characteristic length scales above and below the screen. Now, applying these pressure and length scales to (9)-(12), yields, after removing the bars, the system

$$
\begin{aligned}
\left(H-\frac{\varepsilon^{2}}{\delta} h\right)_{t}-\left[\frac{\left(H-\frac{\varepsilon^{2}}{\delta} h\right)^{3} p_{a x}}{12}-\frac{\left(H-\frac{\varepsilon^{2}}{\delta} h\right)}{2}\right]_{x}+\frac{\lambda \tau \varepsilon^{2}}{\delta} h_{x x} & =0,(17) \\
h_{t}+\left[-\frac{1}{12} h^{3} p_{b x}\right]_{x}-\lambda \tau h_{x x} & =0,(18) \\
\tau h_{x x}-\left[\frac{\varepsilon^{4}}{\delta^{2}} p_{a}-p_{b}\right] & =0,(19) \\
H-\left[\varepsilon^{2}+\frac{1}{2}\left(x-x_{i}-t\right)^{2}\right] & =0,(20)
\end{aligned}
$$

for $0 \leq x \leq 1$

In $(17)-(20)$ we have introduced the four non-dimensional parameters

$$
\varepsilon=\sqrt{\frac{H_{a}}{\kappa_{s}}} \frac{1}{L}, \quad \delta=\frac{H_{a}}{h_{f}}, \quad \tau=\frac{T h_{f}^{3}}{\mu U L^{3}}, \quad \lambda=\frac{\Lambda \mu L^{2}}{h_{f}^{3}} .
$$

Here $\varepsilon(\ll 1)$ is the ratio of the length-scale of the squeegee tip to the length-scale of the screen, and $\delta(\ll 1)$ is the ratio of the squeegee tip height to the unperturbed screen height. The remaining non-dimensional parameters are $\tau$, a measure of the screen tension and $\lambda$, a measure of the permeability of the screen.

Finally, the non-dimensional boundary conditions are

$$
\begin{aligned}
& p_{a}(0, t)=0, \quad p_{b x}(0, t)=0, \quad p_{a}(1, t)=0, \quad p_{b x}(1, t)=0, \\
& h(0, t)=1, \quad h\left(x_{L}, t\right)=H\left(x_{L}, t\right), \quad h_{x}\left(x_{L}, t\right)=H_{x}\left(x_{L}, t\right), \\
& h(1, t)=1, \quad h\left(x_{R}, t\right)=H\left(x_{R}, t\right), \quad h_{x}\left(x_{R}, t\right)=H_{x}\left(x_{R}, t\right),
\end{aligned}
$$


Table I. Typical orders of magnitude for dimensional screen-printing parameters.

\begin{tabular}{lcl}
\hline Parameter & Symbol & Value \\
\hline Frame height & $h_{f}$ & $10^{-3} \mathrm{~m}$ \\
Paste viscosity & $\mu$ & $10 \mathrm{Pas}$ \\
Screen tension & $T$ & $10^{3} \mathrm{~N} \mathrm{~m}^{-1}$ \\
Screen length & $L$ & $10^{-1} \mathrm{~m}$ \\
Squeegee velocity & $U$ & $10^{-1} \mathrm{~m} \mathrm{~s}^{-1}$ \\
Squeegee tip height & $H_{a}$ & $10^{-4} \mathrm{~m}$ \\
Squeegee tip curvature & $\kappa_{s}$ & $10^{3} \mathrm{~m}^{-1}$ \\
\hline
\end{tabular}

and the non-dimensional initial conditions are

$$
h= \begin{cases}1-\frac{\delta}{\varepsilon^{2}}\left(x_{i}-x_{L}\right) & 0<x<x_{L}, \\ \delta+\frac{1}{2} \frac{\delta}{\varepsilon^{2}}\left(x-x_{i}\right)^{2} & x_{L}<x<x_{R}, \\ 1+\frac{\delta}{\varepsilon^{2}}(x-1)\left(x_{R}-x_{i}\right) & x_{R}<x<1,\end{cases}
$$

where

$$
\begin{aligned}
& x_{L}=\sqrt{x_{i}^{2}+2 \frac{\varepsilon^{2}}{\delta}(\delta-1)}, \\
& x_{R}=1-\sqrt{\left(1-x_{i}\right)+2 \frac{\varepsilon^{2}}{\delta}(\delta-1)} .
\end{aligned}
$$

\subsection{PARAMETER VAlues FOR INDUSTRIAL SCREEN-PRINTING}

To proceed further, we now decide upon the parameter sizes most relevant to the industrial screen-printing process. Approximate values of screen-printing quantities are given in Table I. To estimate the screen permeability $\Lambda$, we note that, if the print is to be successful, the timescale for fluid transfer through the screen should be of the same order as that for the squeegee motion. Thus we have

$$
\Lambda=\mathcal{O}\left(\frac{U L}{T}\right)=\mathcal{O}\left(10^{-5}\right) \mathrm{ms}^{-1} \mathrm{~Pa}^{-1} .
$$

Now we use the approximate parameter values for industrial screenprinting to justify our assumption in the tangential boundary condition (3) that the tangential velocity is continuous across the screen. 


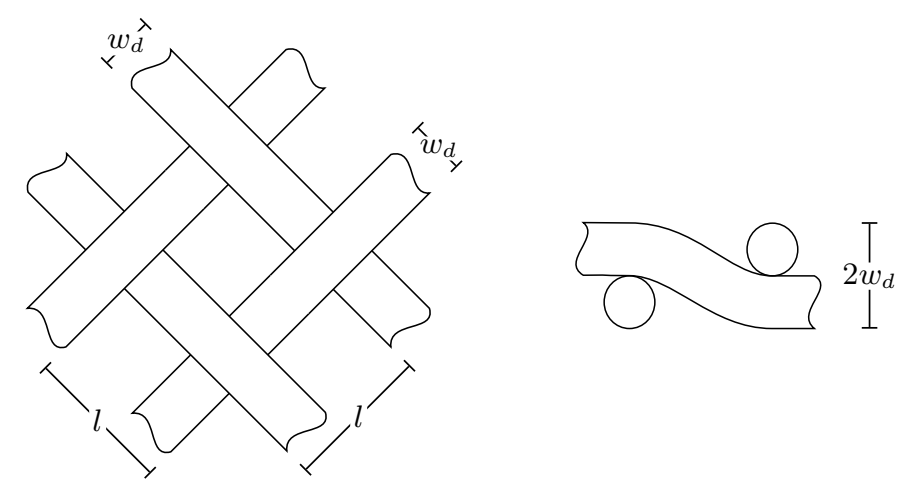

Figure 3. A sketch of the overhead (LHS) and a $45^{\circ}$ cross-section (RHS) of the steel wire cloth used for screen printing. The cloth is oriented for printing from left to right or from top to bottom.

\begin{tabular}{|c|c|c|}
\hline Parameter & Symbol & Value \\
\hline Open area fraction & $\phi$ & 0.44 \\
\hline Wire diameter & $w_{d}$ & $0.5 \times 10^{-4} \mathrm{~m}$ \\
\hline Wire separation & $l$ & $1.5 \times 10^{-4} \mathrm{~m}$ \\
\hline
\end{tabular}

The selection of appropriate boundary conditions for flow past permeable membranes has been the subject of much research. For certain canonical problems, authors including Tio and Sadhal [15] and Pozrikidis $[16,17,18]$ have demonstrated the existence of a macroscopic slip velocity proportional to the difference in shear stress on either side of the membrane. For a membrane modelled as a zerothickness plate perforated by parallel slits separated by a distance $l$, Tio and Sandhal [15] obtained the expression

$$
U_{S}=-\frac{\gamma l}{4 \pi} \log \left[\cos \left(\frac{\phi \pi}{2}\right)\right]
$$

for the slip velocity $U_{S}$, where $\phi$ is the open area fraction and $\gamma$ is the local shear rate. Pozrikidis [18] later showed that a finite thickness does not alter the order of magnitude of the slip velocity, though it may dramatically reduce the drift velocity (the velocity induced on one side of a membrane by a shear flow on the other).

The woven structure of steel screen-printing cloth is illustrated in Figure 3, and typical associated parameter values are given in Table II. 
Although this differs from the canonical membrane structure considered in [15], we can use (27) to estimate the order of magnitude of the slip velocity in industrial screen-printing. We identify $l$ with the wire separation and estimate the shear rate as $\gamma \approx U / h_{f}$ to obtain

$$
\frac{U_{S}}{U} \approx 0.02\left(\frac{l}{h_{f}}\right) \approx 0.003 .
$$

This estimate suggests that the slip velocity is indeed negligible compared to the screen velocity except perhaps close to the contact points between the screen and squeegee where we would expect the shear rate to be large.

\subsection{A Distinguished PARAMETER Limit}

At this stage, we use the industrial screen-printing parameters from Table I (along with the assumption that $H_{a}$ is approximately the print thickness) to find the relative orders of magnitude for $\lambda, \tau, \varepsilon$ and $\delta$ :

$$
\begin{array}{ll}
\varepsilon=\sqrt{\frac{H_{a}}{\kappa_{s}}} \frac{1}{L} \approx 10^{-3}, \quad \delta=\frac{H_{a}}{h_{f}} \approx 10^{-1}, \\
\lambda=\frac{\Lambda \mu L^{2}}{h_{f}^{3}} \approx 10^{3}, \quad \tau=\frac{T h_{f}^{3}}{\mu U L^{3}} \approx 10^{-3} .
\end{array}
$$

We choose to base our asymptotic expansions on the small parameter $\varepsilon$. The orders of magnitude shown in (29) provide a clear indication that the asymptotic regime $\tau=\mathcal{O}(\varepsilon)$ and $\lambda=\mathcal{O}\left(\varepsilon^{-1}\right)$ gives the best model of industrial screen-printing.

Finally, we need to decide upon an appropriate order of magnitude for $\delta$ with respect to $\varepsilon$. If we restrict $\delta$ to lie within the physically reasonable range $\mathcal{O}(\varepsilon) \lesssim \delta \lesssim \mathcal{O}(1)$, then the richest asymptotic limit for (17)-(20) is $\delta=\mathcal{O}(\varepsilon)$. A further justification for this choice is that it is consistent with the use of the lubrication approximation to describe the fluid flow on the length- and height-scales of the tip.

Thus, we set

$$
\delta=\delta_{0} \varepsilon, \quad \tau=\tau_{0} \varepsilon, \quad \lambda=\lambda_{0} \varepsilon^{-1},
$$

where $\lambda_{0}, \tau_{0}$ and $\delta_{0}$ are all $\mathcal{O}(1)$. Substituting the distinguished parameter limits (30) into the outer problem (17)-(20) yields the system

$$
\left(H-\frac{\varepsilon}{\delta_{0}} h\right)_{t}-\left[\frac{\left(H-\frac{\varepsilon}{\delta_{0}} h\right)^{3} p_{a x}}{12}-\frac{\left(H-\frac{\varepsilon}{\delta_{0}} h\right)}{2}\right]_{x}+\frac{\lambda_{0} \tau_{0} \varepsilon}{\delta_{0}} h_{x x}=0
$$




$$
\begin{aligned}
h_{t}-\left[\frac{1}{12} h^{3} p_{b x}\right]_{x}-\lambda_{0} \tau_{0} h_{x x} & =0,(32) \\
\varepsilon \tau_{0} h_{x x}-\left[\frac{\varepsilon^{2}}{\delta_{0}^{2}} p_{a}-p_{b}\right] & =0,(33) \\
H-\left[\varepsilon^{2}+\frac{1}{2}\left(x-x_{i}-t\right)^{2}\right] & =0
\end{aligned}
$$

\section{Leading-order equations}

\subsection{OUter PROBLEM}

We take the limit $\varepsilon \rightarrow 0$ in (31)-(34) to obtain the leading-order equations

$$
\begin{aligned}
H_{t}-\left[\frac{1}{12} H^{3} p_{a x}-\frac{1}{2} H\right]_{x} & =0, \\
h_{t}-\left[\frac{1}{12} h^{3} p_{b x}\right]_{x}-\lambda_{0} \tau_{0} h_{x x} & =0, \\
p_{b} & =0, \\
H-\frac{1}{2}(x-s(t))^{2} & =0,
\end{aligned}
$$

where we have introduced the notation $s(t)=x_{i}+t$ to denote the position of the squeegee tip.

From (35), we find that

$$
p_{a x}=-\frac{12 A_{0}}{H^{3}}-\frac{6}{H^{2}},
$$

where the arbitrary function $A_{0}(t)$ corresponds to the leading-order flux beneath the squeegee. Matching to an inner solution valid near the squeegee tip at $x=s(t)$ (see Section 4.2) requires $A_{0}=0$. Then the leading-order pressure above the screen is

$$
p_{a}= \begin{cases}\frac{2 \sqrt{2}}{H^{3 / 2}}-\frac{8}{s(t)^{3}} & \text { for } 0 \leq x \leq s(t), \\ \frac{2 \sqrt{2}}{H^{3 / 2}}-\frac{8}{(1-s(t))^{3}} & \text { for } s(t) \leq x \leq 1,\end{cases}
$$

where we have applied the boundary conditions (22). From (36) and (37), we find that the screen height obeys the heat equation

$$
h_{t}-\lambda_{0} \tau_{0} h_{x x}=0
$$


with boundary and initial conditions

$$
\begin{gathered}
h(0, t)=1, \quad h(1, t)=1, \quad h(s(t), t)=0, \\
h(x, 0)=\left\{\begin{array}{l}
1-\frac{x}{x_{i}}, \text { for } 0<x<s(t), \\
\frac{x-x_{i}}{1-x_{i}}, \text { for } s(t)<x<1 .
\end{array}\right.
\end{gathered}
$$

It is interesting to contrast (41a) with the well-known lubrication equation

$$
h_{t}+\left(h^{3} h_{x x x}\right)_{x}=0
$$

which governs surface-tension-driven thin-film flow on an impermeable substrate [19]. In our model, there is no leading-order pressure gradient below the screen to drive the flow of paste horizontally. Instead, the paste flows principally through the screen, at a rate proportional to the pressure difference which is itself proportional to the screen curvature. This link between the pressure and curvature is manifested as the product of the non-dimensional permeability and tension, $\lambda_{0} \tau_{0}$, in (41a). Henceforth, we denote the effective diffusion coefficient in (41a) by

$$
D=\lambda_{0} \tau_{0}=\lambda \tau=\frac{\Lambda T}{U L} .
$$

We present numerical and asymptotic solutions of (41) in Sections 5, 6 and 7.

\subsection{INNER PROBLEM CLOSE TO THE SQUEEGEE TIP}

In the outer problem described above, the squeegee acts at a single point, where it forces the screen to make contact with the substrate. In addition, the pressure gradient and, hence, the horizontal flux below the screen are zero to leading order. This outer solution therefore fails to capture the important characteristics of the behaviour near the squeegee tip. For example, as pointed out in Section 3, the squeegee actually makes contact with the screen on a region between two contact points $x_{L}$ and $x_{R}$. For $x \in\left(x_{L}, x_{R}\right)$ it is impossible for paste to flow between the squeegee and the screen, so the flux beneath the screen must become significant.

We therefore examine a boundary layer, in which the geometry of the squeegee tip is resolved, by rescaling as follows:

$$
x=x_{i}+t+\varepsilon x^{\prime}, \quad h=\varepsilon \delta_{0} h^{\prime}, \quad H=\varepsilon^{2} H^{\prime}, \quad p_{a}=\frac{1}{\varepsilon^{3}} p_{a}^{\prime}, \quad p_{b}=\frac{1}{\varepsilon \delta_{0}^{2}} p_{b}^{\prime} .
$$


Substituting these inner scalings into (17)-(18) yields the inner system (after removing the primes)

$$
\begin{aligned}
\varepsilon^{2}(H-h)_{t}-\varepsilon\left[\frac{1}{12}(H-h)^{3} p_{a x}+\frac{1}{2}(H-h)\right]_{x}+D h_{x x} & =0, \\
\varepsilon^{2} h_{t}-\varepsilon\left[\frac{1}{12} h^{3} p_{b x}+h\right]_{x}-D h_{x x} & =0, \\
\varepsilon \tau_{0} h_{x x}-\frac{1}{\delta_{0}^{3}}\left(p_{a}-p_{b}\right) & =0, \\
H-\left[1+\frac{1}{2} x^{2}\right] & =0 .
\end{aligned}
$$

Now, in the limit $\varepsilon \rightarrow 0$, we obtain

$$
p_{a}-p_{b}=0,
$$

that is, the pressures above and below the screen are equal (though their value is not constant with respect to space and time), which implies that the leading-order flux through the screen is zero in this region. From (45), we obtain $h_{x x}=0$, so that $h$ is linear in $x$ wherever there is no contact with the squeegee. It follows that

$$
h= \begin{cases}x_{L} x+\left(1-\frac{1}{2} x_{L}^{2}\right) & x<x_{L}, \\ 1+\frac{1}{2} x^{2} & x_{L}<x<x_{R}, \\ x_{R} x+\left(1-\frac{1}{2} x_{R}^{2}\right) & x_{R}<x\end{cases}
$$

where $x_{L}$ and $x_{R}$ are the left and right points where the screen and squeegee come into contact.

To determine the pressures, we add (45) and (46) to obtain, to lowest order, the equation

$$
\left[-\frac{1}{12}\left[h^{3}+(H-h)^{3}\right] p_{b x}-\frac{1}{2}(H+h)\right]_{x}=0,
$$

which is valid in regions with and without contact between the squeegee and the screen. We solve (51) explicitly for $p_{b x}$ giving

$$
p_{b x}= \begin{cases}\frac{-12 A_{L}-6(H+h)}{h^{3}+(H-h)^{3}} & x<x_{L}, \\ -\frac{12\left(A_{M}+h\right)}{h^{3}} & x_{L}<x<x_{R}, \\ \frac{-12 A_{R}-6(H+h)}{h^{3}+(H-h)^{3}} & x_{R}<x,\end{cases}
$$


where $A_{L}(t), A_{M}(t)$ and $A_{R}(t)$ represent the flux in each of the three respective regions. To ensure that the flux is conserved as we pass between neighbouring domains, we must have

$$
A_{L}=A_{M}=A_{R} \equiv A \text {. }
$$

We integrate (52) again to obtain the pressure below the screen as

$$
p_{b}= \begin{cases}-A I_{1}\left(-x ;-x_{L}\right)-I_{2}\left(-x ;-x_{L}\right)+B_{L} & x<x_{L}, \\ A F_{M}(x)+G_{M}(x)+B_{M} & x_{L}<x<x_{R}, \\ A I_{1}\left(x ; x_{R}\right)+I_{2}\left(x ; x_{R}\right)+B_{R} & x_{R}<x,\end{cases}
$$

where

$$
\begin{aligned}
& I_{1}(x ; \xi)=\int_{x}^{\infty} \frac{96 \mathrm{~d} z}{\left(2 \xi z+2-\xi^{2}\right)^{3}+(z-\xi)^{6}}, \\
& I_{2}(x ; \xi)=\int_{x}^{\infty} \frac{24\left(4+(z+\xi)^{2}\right) \mathrm{d} z}{\left(2 \xi z+2-\xi^{2}\right)^{3}+(z-\xi)^{6}}, \\
& F_{M}(x)=-\frac{3 x\left(10+3 x^{2}\right)}{\left(2+x^{2}\right)^{2}}-\frac{9}{\sqrt{2}} \tan ^{-1}\left(\frac{x}{\sqrt{2}}\right), \\
& G_{M}(x)=-\frac{12 x}{2+x^{2}}-6 \sqrt{2} \tan ^{-1}\left(\frac{x}{\sqrt{2}}\right),
\end{aligned}
$$

and $B_{L}(t), B_{M}(t)$ and $B_{R}(t)$ represent pressures, which will be chosen to satisfy the boundary conditions.

Continuity of the pressures at the left and right contact points leads to

$$
\begin{aligned}
- & A I_{1}\left(-x_{L} ;-x_{L}\right)-I_{2}\left(-x_{L} ;-x_{L}\right)+B_{L}=A F_{M}\left(x_{L}\right)+G_{M}\left(x_{L}\right)+B_{M}, \\
& A I_{1}\left(x_{R} ; x_{R}\right)+I_{2}\left(x_{R} ; x_{R}\right)+B_{R}=A F_{M}\left(x_{R}\right)+G_{M}\left(x_{R}\right)+B_{M} .
\end{aligned}
$$

To close the system, we require four more conditions since the contact points $x_{L}$ and $x_{R}$ are also unknown a priori.

\subsection{Matching}

To close our inner problem we must match the solutions for the pressures and the screen gradients to those of the outer problem. For clarity, we re-introduce bars to denote outer variables and primes to denote inner ones. The matching conditions for $\bar{h}$ are

$$
\bar{h}_{\bar{x}}\left(\bar{x}_{i}+\bar{t}, \bar{t}\right) \rightarrow\left\{\begin{array}{l}
\delta_{0} x_{L} \text { as } \bar{x} \rightarrow\left(\bar{x}_{i}+\bar{t}\right)^{-} \\
\delta_{0} x_{R} \text { as } \bar{x} \rightarrow\left(\bar{x}_{i}+\bar{t}\right)^{+}
\end{array},\right.
$$


while, for the inner pressure, we obtain

$$
\lim _{x^{\prime} \rightarrow \pm \infty} p_{b}^{\prime}\left(x^{\prime}, t^{\prime}\right)=0 .
$$

Once $\bar{h}$ has been found by solving the outer problem (41), the contact points $x_{L}$ and $x_{R}$ are determined by (57). From (58) it follows that $B_{L}=B_{R}=0$, so the flux $A$ is given by the equation

$$
A=\frac{I_{2}\left(-x_{L} ;-x_{L}\right)+G_{M}\left(x_{L}\right)-G_{M}\left(x_{R}\right)+I_{2}\left(x_{R} ; x_{R}\right)}{I_{1}\left(-x_{L} ;-x_{L}\right)+F_{M}\left(x_{L}\right)-F_{M}\left(x_{R}\right)+I_{2}\left(x_{R} ; x_{R}\right)} .
$$

\section{Numerical results}

We now show numerical solutions of two systems, chosen to be representative of the different behaviours possible with our model. In one system we take $D=0.01$ (low-permeability regime) while in the other we take $D=1$ (high-permeability regime); in all other respects, the systems are identical. The squeegee is initially at $x_{i}=0.3$ and we observe the behaviour for $t \in[0,0.4]$, with $\delta_{0}=1$.

We obtain the numerical solutions for the outer region in a timedependent coordinate system which maps the regions $x \in[0, s(t)]$ and $x \in[s(t), 1]$ to the unit interval. In this new coordinate system (described below in Section 7), (41) is solved for $h(x, t)$ using the Crank-Nicholson scheme. The matching condition (57) then enables us to find $x_{L}$ and $x_{R}$ and thus obtain the inner solution for the screen. To solve for the pressure in the inner region, we evaluate the integrals in (54) numerically, using adaptive Gaussian quadrature.

\subsection{SCREEN DEFLECTION IN OUTER REGION}

The deflection of the screen in the outer region for both values of $D$ is illustrated in Figure 4. The behaviours of the two systems are markedly different. The screen profile is approximately linear in the high-permeability regime while, in the low-permeability regime, the following features consistent with industrial observations appear. There is a sharp deflection ahead of the squeegee as the fluid piles up, unable to move easily through the screen. Behind the squeegee, the screen sticks to the substrate before 'peeling' off some time after the squeegee has passed. Since the second regime reproduces much of the qualitative behaviour observed in practice, it will likely have the greatest industrial relevance. 

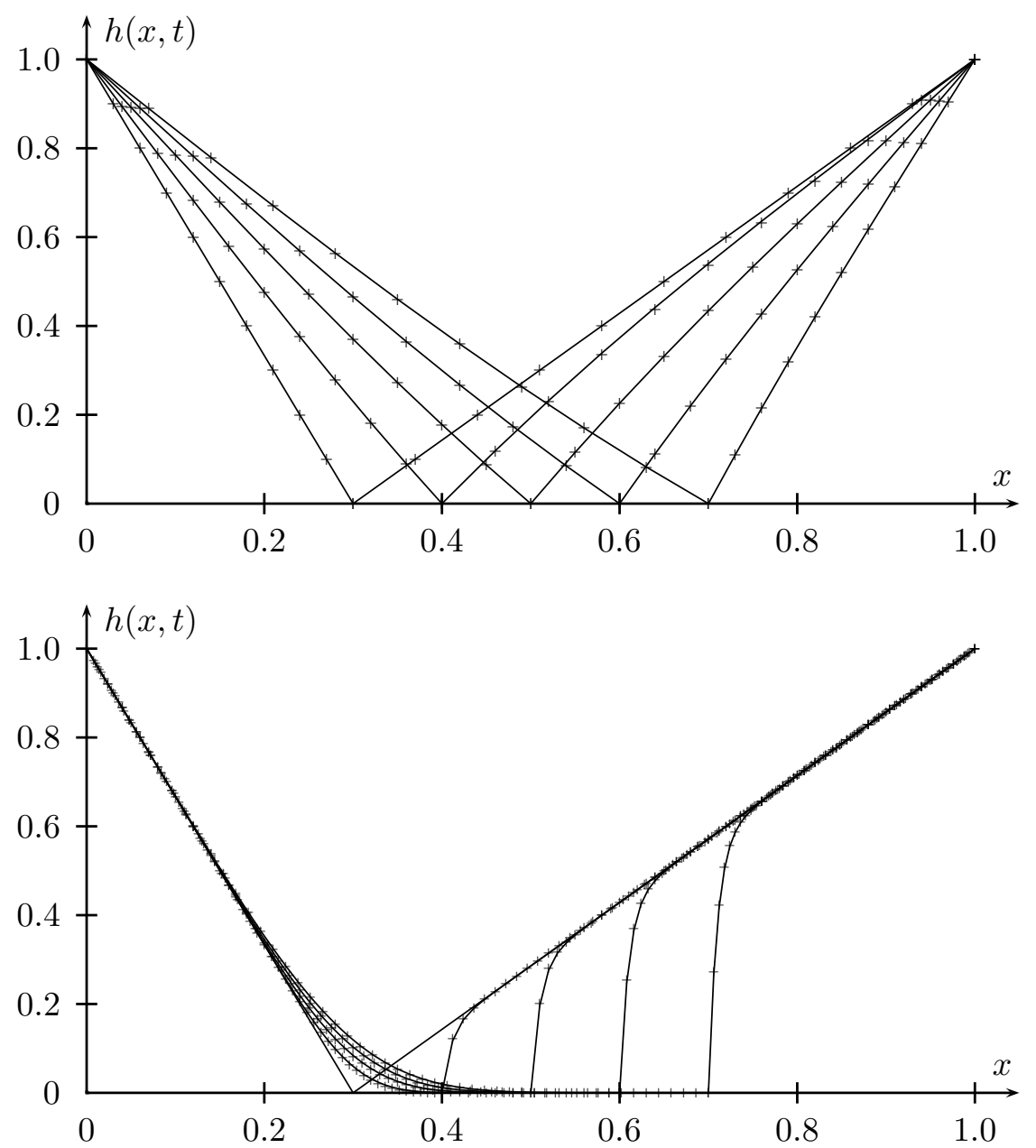

Figure 4. Screen deflection in the outer region for $D=1$ (top) and $D=0.01$ (bottom) for the times $t=\{0,0.1,0.2,0.3,0.4\}$. Numerical results are shown by ' + ' marks while asymptotic results (see Section 5 ) are shown by solid lines.

\subsection{Flux through the SCREen in the outer Region}

The dimensionless flux through the screen (measured in the upward direction) is given by

$$
q(x, t)=-D h_{x x} .
$$

The flux in the outer region is shown in Figure 5, where the differences between the two systems are again apparent. For $D=0.01$, the flow through the screen is restricted to a small region in front of the squeegee and, in this region, we observe that the paste flows up through the screen. Intuitively, we would expect the flow to be in this direction to 

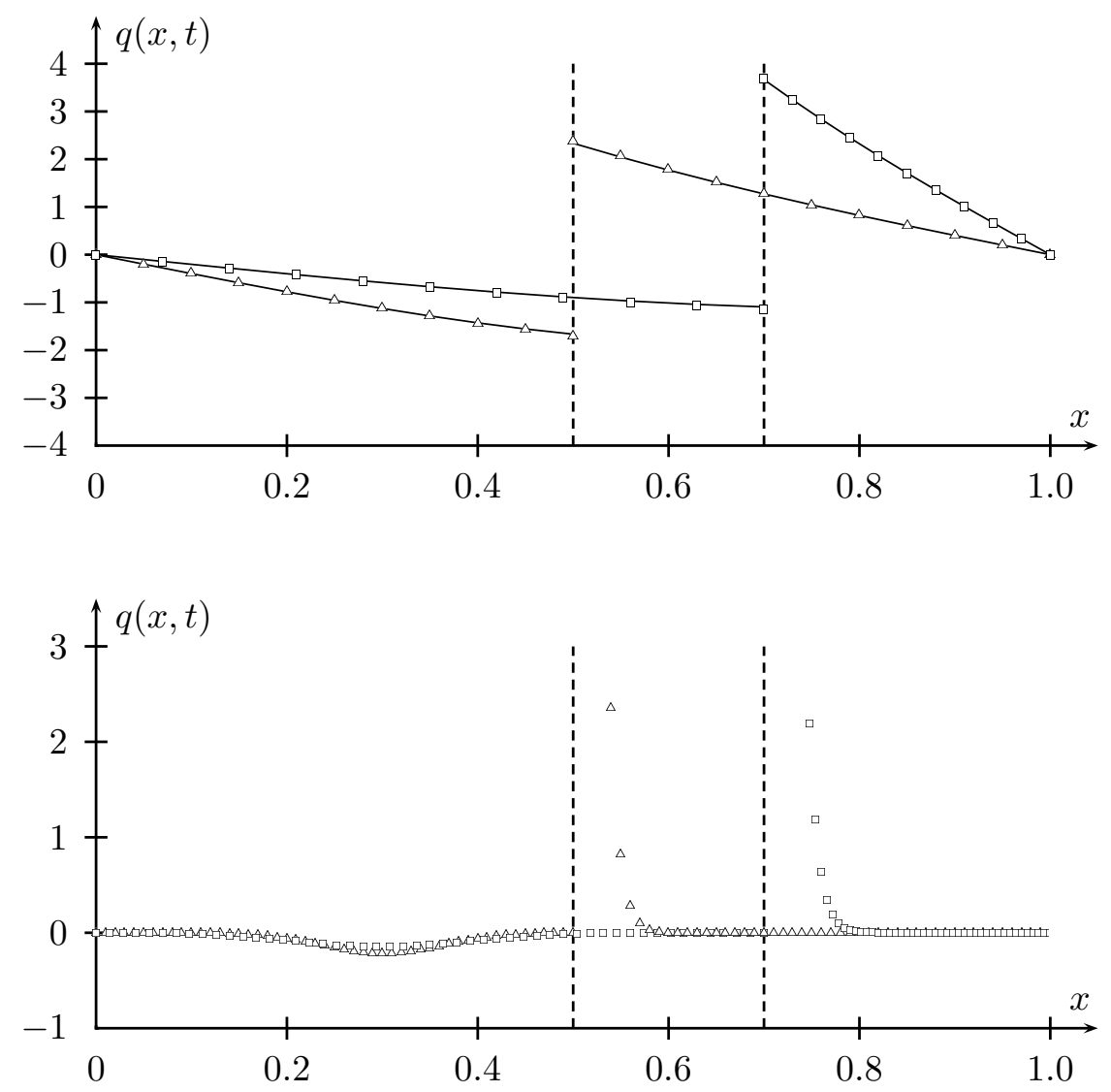

Figure 5. Flux through the screen in the outer region for $D=1$ (top) and $D=0.01$ (bottom). Numerical results for $t=0.2,0.4$ are shown by triangles and squares respectively, while asymptotic results (see Section 5) are shown by solid lines. The squeegee's position at these times is indicated by the vertical dashed lines.

conserve mass as the area below the screen in front of the squeegee is reduced. In the high-permeability limit, the flow of paste through the screen is no longer localised near the squeegee. Again, paste flows up as the screen is squeezed ahead of the squeegee and back down through the screen as it relaxes behind the squeegee.

\subsection{Contact point motion}

In Figure 6, we show the evolution of the squeegee/screen contact points. In the system with $D=1$, we observe that the contact points 'roll' forward at a similar rate. A consequence of this is that the length of screen in contact with the squeegee does not change appreciably 

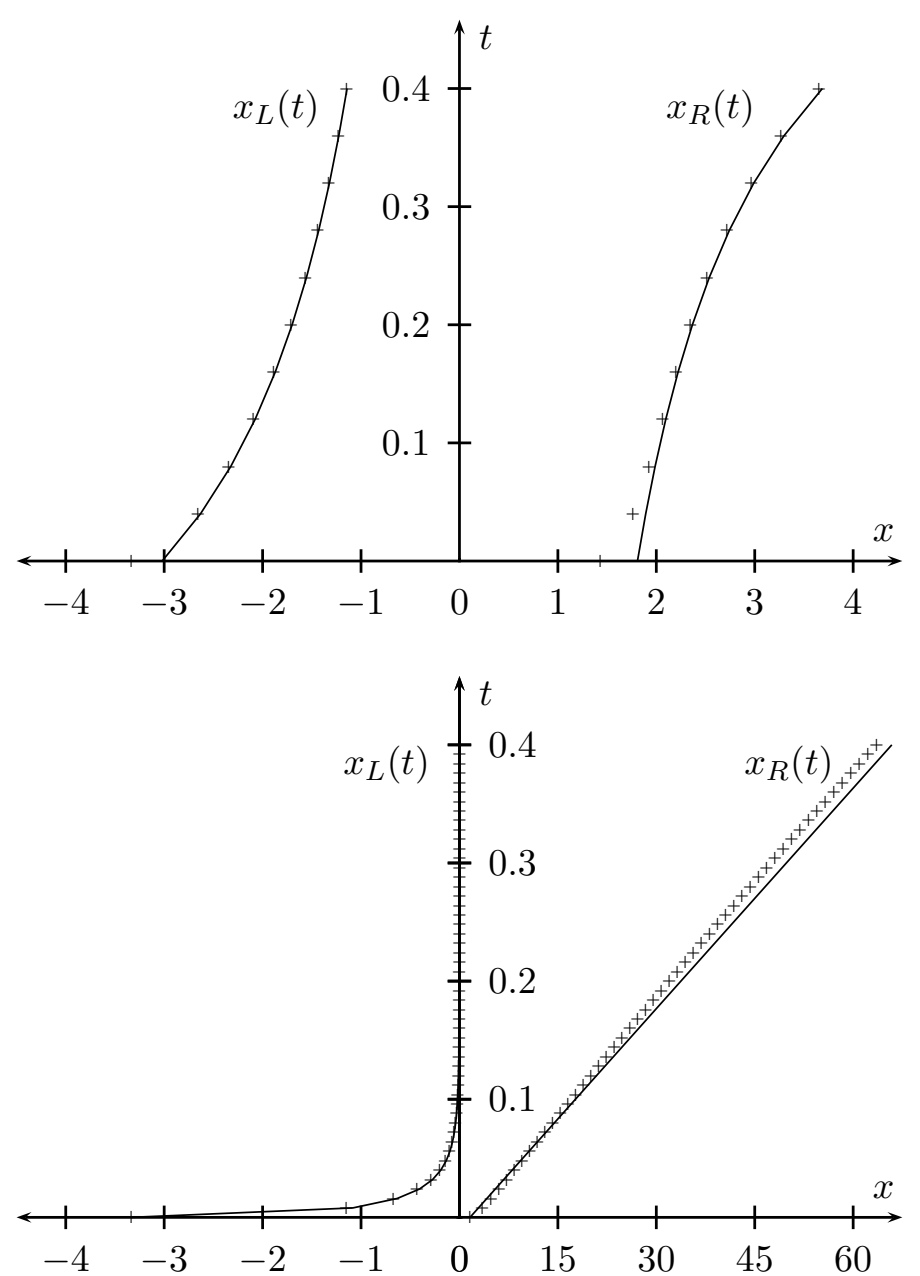

Figure 6. Contact points for the squeegee and screen in the outer region for $D=1$ (top) and $D=0.01$ (bottom). Numerical results for $t=0.2,0.4$ are shown by triangles and squares respectively, while asymptotic results (see Section 5) are shown by solid lines. Note that (i) the scale on the x-axis is different on either side of the origin for $D=0.01$, and (ii) the first two terms in the asymptotic expansion for $x_{L}$ and $x_{R}$ were used to plot the asymptotic result for $D=1$.

over the print. However, when $D=0.01$, the rear contact point moves quickly to the tip of the squeegee, while the front contact point moves forward at near-constant speed. Thus, the length of the screen in contact with the squeegee continually increases throughout the print. Since paste can only flow through the screen where there is no contact, this suggests that, as a print progresses, transfer of paste occurs increasingly far ahead of the tip. 


\subsection{Flux through the SCREen IN THE INNER REGion}

The flux of paste through the screen in the inner region, given by

$$
q=\delta_{0}\left[\frac{1}{12} h^{3} p_{b x}+h\right]_{x}=\delta_{0}\left[h-\frac{h^{3}}{2}\left(\frac{2 A+H+h}{h^{3}+(H-h)^{3}}\right)\right]_{x},
$$

is plotted in Figure 7. As a verification of the numerical calculations, the value at infinity in the inner region in either case matches to the flux evaluated at the squeegee tip in the outer region as shown in Figure 5.

Ahead of the contact region (where the flux through the screen is necessarily zero), there is a small region in which fluid is transfered down through the screen, and printing would therefore take place. The flux then increases, achieving a maximum before levelling off to a uniform value at infinity. In the high-permeability regime, the flux profile displays a high degree of symmetry while, in the low-permeability regime, a much greater flux is achieved ahead of the squeegee than behind, and continues to grow throughout the print. This suggests that more effective printing may be achieved as the squeegee approaches $x=1$.

\subsection{PRESSURE IN THE INNER REGION}

In the inner region, we need not distinguish the pressures above and below the screen since they are equal to lowest order. The pressure profile is plotted in Figure 8. In both regimes, the pressure increases as the paste is squeezed ahead of the squeegee and decreases behind. The pressure profile has roughly the same magnitude and shape in either regime and changes little throughout the print run. This demonstrates that the dramatic dependence on $D$ shown in Figures 4-7 is not caused by a large change in the pressure but, rather, by a dramatically different response of the screen to approximately the same pressure field.

\section{Asymptotic solution for $D \ll 1$.}

Now we use asymptotic analysis to provide validation and interpretation of the numerical results shown above. We begin with the lowpermeability limit $D \ll 1$, in which the flow of paste through the screen is principally confined to a neighbourhood of the squeegee where the pressure and screen curvature are largest.

To lowest order in $D,(41 \mathrm{a})$ has the solution $h(x, t) \sim h(x, 0)$. However, this solution fails to satisfy the boundary condition that $h$ must be zero at $x=s(t)$, indicating the presence of a boundary layer just 

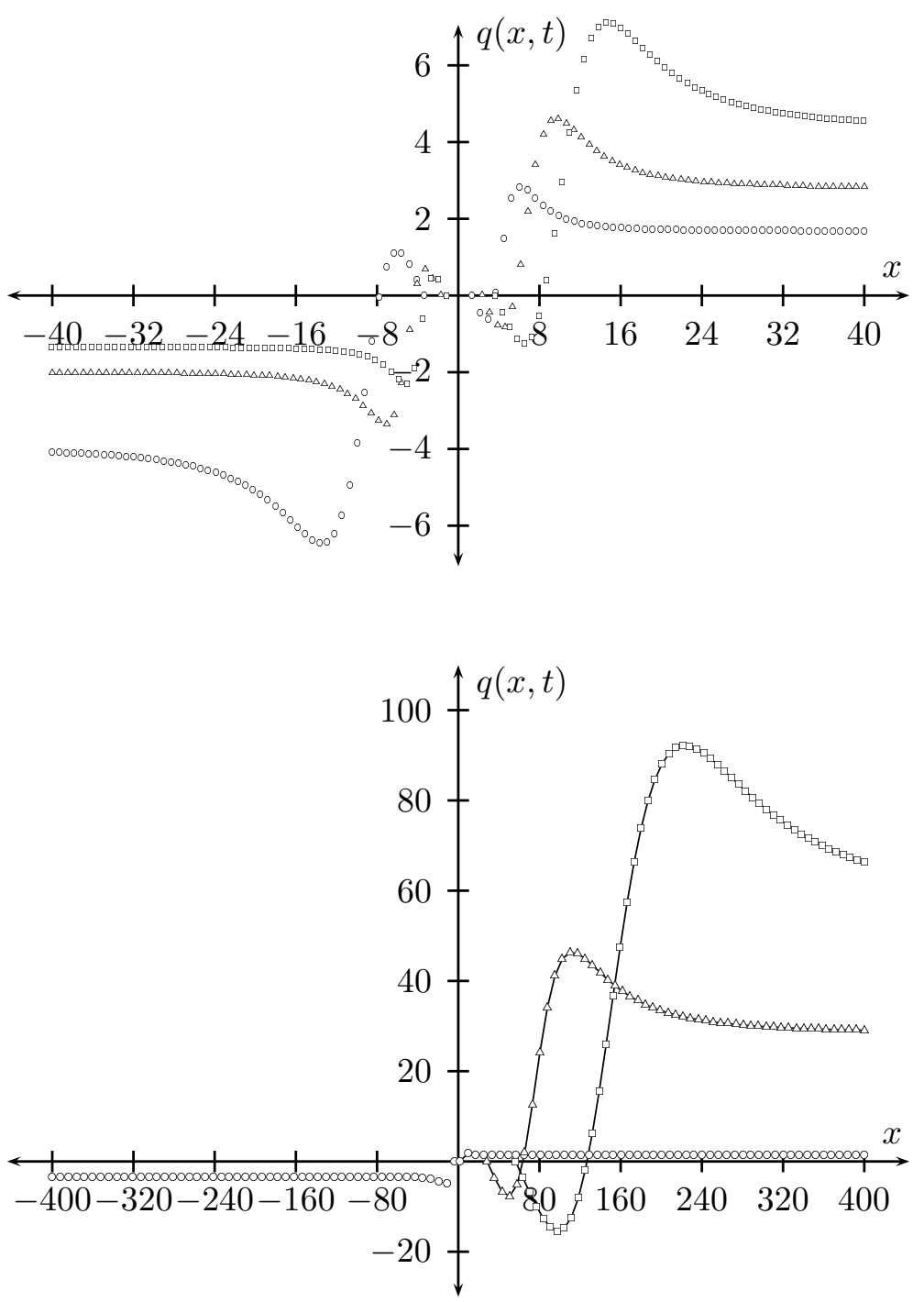

Figure 7. Flux through the screen in the inner region for $D=1$ (top) and $D=0.01$ (bottom). Numerical results for $t=0,0.2,0.4$ are shown by circles, triangles and squares respectively, while asymptotic results (see Section 5) are shown by solid lines. 

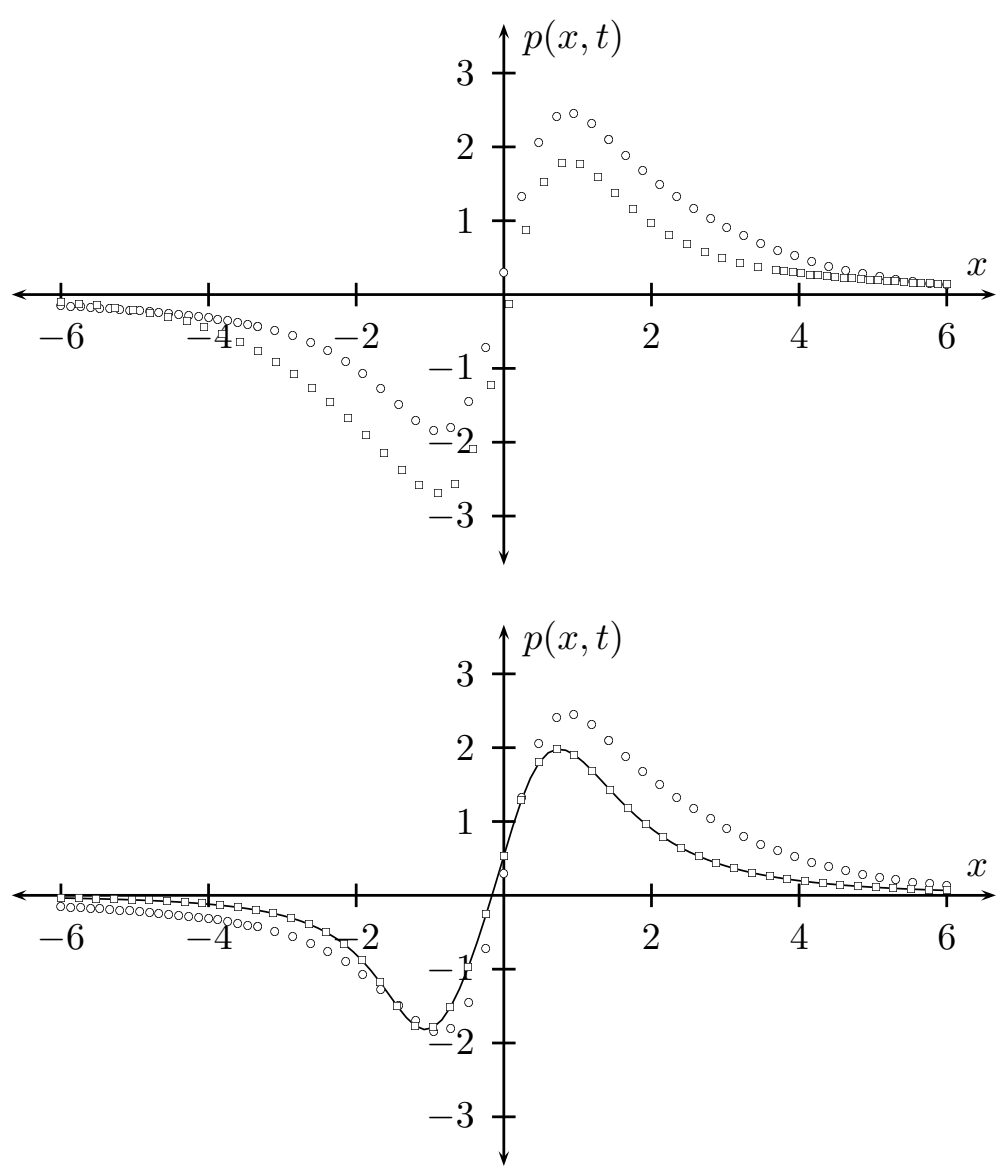

Figure 8. Pressure in the paste in the inner region for $D=1$ (top) and $D=0.01$ (bottom) for $t=0$ (circles) and $t=0.4$ (squares). The asymptotic result for $\mathrm{D}=0.01$ (see Section 5 ) is shown by a solid line.

ahead of the squeegee. To investigate this boundary layer, we apply the transformation $(x, t) \rightarrow(\eta, t)$, where $x=s(t)+D \eta$, to (41a), obtaining

$$
D h_{t}-h_{\eta}-h_{\eta \eta}=0 .
$$

To lowest order in $D$, this has the solution

$$
h \sim \frac{t}{1-x_{i}}\left(1-\mathrm{e}^{-\eta}\right) \quad \eta>0,
$$

where we have applied the boundary condition $h(0, t)=0$ and the matching condition

$$
\lim _{\eta \rightarrow \infty} h(\eta, t)=\frac{t}{1-x_{i}} .
$$


The implication of this solution is that the screen height rapidly increases from zero to its undeformed height in a small region, of width $\mathcal{O}(D)$, just ahead of the squeegee. The composite expansion

$$
h(x, t) \sim \frac{x-x_{i}}{1-x_{i}}-\frac{t}{1-x_{i}} \exp \left(\frac{s(t)-x}{D}\right), \quad x>s(t)
$$

is plotted along with our numerical solution in Figure $4 \mathrm{~b}$, where we see that they agree extremely well.

From (63) and (57) we can read off the asymptotic behaviour of $x_{R}$ as

$$
x_{R} \sim \frac{t}{D \delta_{0}\left(1-x_{i}\right)}+\frac{1}{\delta_{0}\left(1-x_{i}\right)} .
$$

Thus, as seen in Figure 6b, $x_{R}$ increases linearly, becoming of order $1 / D$ over an order one time scale. Likewise, the flux through the screen in this region is given by

$$
q=-D h_{x x}=-\frac{h_{\eta \eta}}{D} \sim \frac{t \mathrm{e}^{-\eta}}{D\left(1-x_{i}\right)},
$$

which is of order $1 / D$ and confined to a small region ahead of the squeegee (of length $\mathcal{O}(1 / D)$ ), in agreement with Figure 5b.

We now turn our attention to the outer problem behind the squeegee, where (41a) again implies that $h$ is time-independent to lowest order in $D$. Now we have $h(s(t), t)=0$ as well as the initial condition, so the leading-order solution as $D \rightarrow 0$ is

$$
h= \begin{cases}1-\frac{x}{x_{i}} & 0<x<x_{i}, \\ 0 & x_{i}<x<s(t) .\end{cases}
$$

Evidently there must be a boundary layer near $x=x_{i}$ where the curvature term in the heat equation becomes significant, and we examine this via the rescaling $x=x_{i}+\sqrt{D} \zeta, h=\sqrt{D} g$. This recovers the heat equation

$$
g_{t}=g_{\zeta \zeta}
$$

subject to the boundary and initial conditions

$$
\begin{aligned}
& g \sim-\frac{\zeta}{x_{i}} \quad \text { as } \zeta \rightarrow-\infty, \\
& g \rightarrow 0 \quad \text { as } \zeta \rightarrow+\infty, \\
& g=\left\{\begin{array}{ll}
-\frac{\zeta}{x_{i}} & \zeta<0, \\
0 & \zeta>0 .
\end{array} \quad \text { at } t=0 .\right.
\end{aligned}
$$


The solution of (69) in original variables is

$$
h=\frac{1}{2}\left(1-\frac{x}{x_{i}}\right) \operatorname{erfc}\left(\frac{\left(x-x_{i}\right)}{2 \sqrt{D t}}\right)+\frac{\sqrt{D t}}{x_{i} \sqrt{\pi}} \exp \left(\frac{-\left(x-x_{i}\right)^{2}}{4 D t}\right),
$$

for $x<s(t)$, which is plotted in Figure 4b. Again, the numerical and asymptotic solutions are very close. The asymptotic solution shows how the screen sticks to the substrate, so that the peeling front, which moves like $x \sim x_{i}+\sqrt{D t}$, lags behind the squeegee at $x=s(t)$.

The asymptotic solution (70) is valid when the squeegee has moved a significant distance from $x_{i}$ and, therefore, fails to capture the initial rapid evolution in $x_{L}$. To investigate the early stages of peeling, we perform a second rescaling, namely $x=s(t)-D X, t=D \hat{t}, h=D \hat{h}$, which transforms the problem to

$$
\begin{aligned}
\hat{h}_{\hat{t}}+\hat{h}_{X} & =\hat{h}_{X X}, \quad X>0, \\
\hat{h}(X, 0)=\frac{X}{x_{i}}, & \hat{h}(0, \hat{t})=0, \quad \hat{h} \sim \frac{X}{x_{i}} \text { as } X \rightarrow \infty .
\end{aligned}
$$

The solution is

$$
\hat{h}=\frac{1}{x_{i}}\left[X-\hat{t}+\frac{X}{2 \sqrt{\pi}} \int_{0}^{\hat{t}} \frac{\hat{t}-\sigma}{\sigma^{3 / 2}} \mathrm{e}^{-(\sigma-X)^{2} / 4 \sigma} \mathrm{d} \sigma\right],
$$

from which we can deduce the slope of the screen at $x=x_{i}$ and hence, using (57), the left-hand contact point:

$$
\begin{aligned}
x_{L} & =\frac{1}{\delta_{0}} h_{x}(s(t), t) \\
& =-\frac{1}{\delta_{0}} \hat{h}_{X}(0, \hat{t}) \\
& =-\frac{1}{\delta_{0} x_{i}}\left[\left(1+\frac{\hat{t}}{2}\right) \operatorname{erfc}\left(\frac{\sqrt{\hat{t}}}{2}\right)-\sqrt{\frac{\hat{t}}{\pi}} \mathrm{e}^{-\hat{t} / 4}\right] .
\end{aligned}
$$

This demonstrates how $x_{L}$ rapidly increases from its initial value of $-1 / \delta_{0} x_{i}$ to 0 , over a time scale of order $D$. In Figure $6 \mathrm{~b}$, we show that (73) successfully predicts the behaviour of $x_{L}$ observed numerically.

The leading-order flux in this region is given by (70) as

$$
q=-D h_{x x}=-\sqrt{\frac{D}{\pi t}} \frac{\mathrm{e}^{-\left(x-x_{i}\right)^{2} / 4 D t}}{2 x_{i}} .
$$

This confirms that the flux behind the squeegee is negative, although small (of order $\sqrt{D / t}$ ) and confined to an $\mathcal{O}(\sqrt{D t}$ ) neighbourhood of $x_{i}$. 
Now, we can use the asymptotic behaviours of $x_{L}$ and $x_{R}$ to estimate the flux constant $A$ given by (59). In the limit $x_{L} \rightarrow 0$ and $x_{R} \rightarrow \infty$, the integrals $I_{1}$ and $I_{2}$ may be evaluated explicitly, leading to

$$
A \sim-\frac{32}{25} \text {. }
$$

On an order-one time-scale, the rate at which fluid flows beneath the squeegee therefore approaches this constant value as $D \rightarrow 0$. By letting $x_{L} \rightarrow 0$ and $x_{R} \rightarrow \infty$ in (61) and applying (75), we estimate the flux through the screen in the inner region as

$$
\frac{q}{\delta_{0}} \sim \begin{cases}\frac{8 x\left(25 x^{6}-42 x^{4}-100\right)}{25\left(8+x^{6}\right)^{2}} & x<x_{L} \\ x_{R} f_{R}\left(x / x_{R}\right) & x>x_{R}\end{cases}
$$

where

$$
\begin{aligned}
f_{R}(y)= & \frac{(1-y)\left(1-y-2 y^{2}+y^{3}\right)}{2 y^{3}\left(3-12 y+15 y^{2}-6 y^{3}+y^{4}\right)^{2}}\{3-24 y \\
& \left.+81 y^{2}-147 y^{3}+150 y^{4}-80 y^{5}+18 y^{6}-2 y^{7}\right\} .
\end{aligned}
$$

Behind the squeegee, the flux is of order one and its profile is approximately time-independent. Ahead of the squeegee, the flux is much larger, with the profile growing and spreading linearly with time (since $x_{R}$ is proportional to $\left.t / D\right)$. Figure $7 \mathrm{~b}$ shows that these predictions are supported by our numerical results.

Now we use (54) to estimate the pressure in the inner region as

$$
p_{b} \sim\left\{\begin{array}{lr}
\frac{3 \pi \sqrt{2}}{25}-2 \sqrt{2} \tan ^{-1}\left(\frac{x^{3}}{2 \sqrt{2}}\right)+\frac{84 x}{25} \mathrm{~F}\left(\frac{1}{6}, 1, \frac{7}{6} ;-\frac{x^{6}}{8}\right) & x<x_{L}, \\
\frac{12 x\left(30-x^{2}\right)}{25\left(2+x^{2}\right)^{2}}+\frac{6 \sqrt{2}}{25} \tan ^{-1}\left(\frac{\sqrt{2}}{x}\right) & x_{L}<x<x_{R}, \\
0 & x>x_{R},
\end{array}\right.
$$

where $\mathrm{F}$ is a hypergeometric function [20, page 1065]. As shown in Figure 8b, our numerical solution agrees well with (78) after an initial transient. We observe that, despite the large flux ahead of the squeegee, the pressure is order one and roughly independent of time. 


\section{Asymptotic solution for $D \gg 1$.}

In Figure 4a we see that, when $D=1$, the screen deflection in the outer region is approximately linear. We now explain this observation by analysing our model asymptotically in the limit $D \rightarrow \infty$, in which the screen is permeable enough to offer little resistance to any paste flowing through it. It is convenient to develop our asymptotic solution in a coordinate system where the squeegee is stationary. This is accomplished with the transformation $(x, t) \rightarrow(\zeta, t)$ where $\zeta \in[0,1]$,

$$
\zeta= \begin{cases}\frac{x}{s(t)} & \text { for } x \in[0, s(t)] \\ \frac{1-x}{1-s(t)} & \text { for } x \in[s(t), 1]\end{cases}
$$

and $s(t)=x_{i}+t$. In this new coordinate system, the equations read

$$
h_{t}-\frac{D}{s^{2}} h_{\zeta \zeta}-\frac{\zeta}{s} h_{\zeta}=0, \quad h(0, t)=1, h(1, t)=0,
$$

for $x \in[0, s]$, and

$$
h_{t}-\frac{D}{(1-s)^{2}} h_{\zeta \zeta}+\frac{\zeta}{(1-s)} h_{\zeta}=0, \quad h(0, t)=1, h(1, t)=0,
$$

for $x \in[s, 1]$.

It is now straightforward to solve for $h$ as an asymptotic expansion in powers of $1 / D$ :

$$
h \sim \begin{cases}1-\zeta-\frac{1}{D}\left[\frac{1}{6} s \zeta\left(1-\zeta^{2}\right)\right]+\mathcal{O}\left(\frac{1}{D^{2}}\right), & x \in[0, s], \\ 1-\zeta+\frac{1}{D}\left[\frac{1}{6}(1-s) \zeta\left(1-\zeta^{2}\right)\right]+\mathcal{O}\left(\frac{1}{D^{2}}\right), & x \in[s, 1] .\end{cases}
$$

This indicates that $h$ is indeed linear in the limit $D \rightarrow \infty$. The leadingorder approximation from (82) is plotted in Figure 4a, and the agreement with our numerical solution is excellent. This verifies the accuracy of our numerical scheme and demonstrates that the series expansions in (82) are rapidly convergent even when $D=1$. For example, the firstorder corrections in $(82)$ are bounded by $s /(9 \sqrt{3} D)$ and $(1-s) /(9 \sqrt{3} D)$ respectively.

Now we can use (60) and (82) to estimate the flux through the screen when $D$ is large:

$$
q=-D h_{x x} \sim \begin{cases}-\frac{x}{s^{2}}, & x \in[0, s] \\ \frac{1-x}{(1-s)^{2}}, & x \in[s, 1] .\end{cases}
$$


This successfully reproduces the roughly linear behaviour observed in Figure 5a and indicates that the flux becomes independent of $D$ in this limit. Similarly, we can use (57) and (82) to obtain the leading-order behaviour of the contact points as

$$
x_{L} \sim-\frac{1}{\delta_{0} s}, \quad x_{R} \sim \frac{1}{\delta_{0}(1-s)} .
$$

As shown in Figure 6a, the contact points follow (84) closely, even when $D=1$.

Since both $x_{L}$ and $x_{R}$ are order one, it is not possible to simplify significantly the inner equations near the squeegee in this regime. Nevertheless, we can explain the symmetry observed in the inner flux and pressure profiles by considering two different squeegee positions $s_{1}$ and $s_{2}$ that are the same distance either side of the midpoint of the screen, that is

$$
s_{1}+s_{2}=1 .
$$

It follows from (84) that the left- and right-hand contact points corresponding to these values of $s$ enjoy the symmetries

$$
x_{L 1}=-x_{R 2}, \quad x_{L 2}=-x_{R 1} .
$$

From these identities and the oddness of the functions $F_{M}(x)$ and $G_{M}(x)$, we deduce that the flux constant $A$ takes the same value when $s=s_{2}$ as it does when $s=s_{1}$. Now (54) and (61) imply that $p_{b}$ and $q$ have the following properties (in the limit $D \rightarrow \infty$ ).

1. In $x_{L}<x<x_{R}$, the pressure profile is the same at $s=s_{1}$ as it is at $s=s_{2}$, but shifted up or down uniformly (by an amount corresponding to $B_{M}$ ).

2. The pressure and flux profiles at $s=s_{1}$ in $x<x_{L}$ are minus those at $s=s_{2}$ in $x>x_{R}$.

That our numerical solutions share these properties may be observed in Figures 7a and 8a.

\section{Conclusions}

We have presented a mathematical model for the screen-printing process, under the assumption that the areas above and below the screen are both flooded with a Newtonian fluid. We identified a parameter regime that appears to be of greatest relevance to industrial screenprinting. In this regime, the flow is decomposed into an outer region, 
in which the squeegee effectively acts at a point and the screen height satisfies the heat equation, and an inner region where the geometry of the squeegee is resolved in detail and the screen deflection is linear. The leading-order equations in these regions were solved numerically and asymptotically, allowing us to determine how the process depends on the physical parameters.

As the paste is displaced by the squeegee, it responds by deforming the screen and by flowing through it. We found that the large-scale behaviour of the screen is hence governed by a single dimensionless group that combines these effects, namely

$$
D=\frac{\Lambda T}{U L},
$$

where $\Lambda, T$ and $L$ are the permeability, tension and length of the screen, while $U$ is the squeegee velocity. This parameter represents an inverse Péclet number, the product $\Lambda T$ having the dimensions of a diffusion coefficient. This effective diffusion models the flow of paste through the screen, driven by a pressure difference which is proportional to the curvature. A similar effect has been observed previously in models for the spreading and imbibition of a viscous liquid on a porous base $[21,22,23]$.

With $D$ small, we obtain behaviours that are familiar within the context of industrial screen-printing and, consequently, we concentrate our discussion on this case. For instance, the screen deforms in a small region immediately in front of the moving squeegee and we observe a peeling motion as the screen lifts up behind the squeegee. We also find that the flux of paste through the screen is very much greater ahead of the squeegee than behind.

From experimental considerations, the value of $T$ is well known but the size of $\Lambda$ is much less certain. By modelling flow through the screen as Poiseuille flow through an array of holes, each of length $2 w_{d}$ and cross-sectional area $A$, we can estimate $\Lambda$ as

$$
\Lambda \approx \frac{\phi A}{16 \pi \mu w_{d}},
$$

where $\phi$ is the open area fraction as before. The typical screen parameters given in Tables I and II give $\Lambda \approx 2 \times 10^{-7} \mathrm{~m} \mathrm{~s}^{-1} \mathrm{~Pa}^{-1}$, which would indeed make $D$ small, of order $2 \times 10^{-2}$, as suggested by our numerical simulations.

We found that the flow near the squeegee tip involves a second dimensionless parameter

$$
\delta_{0}=\frac{L}{h_{f}} \sqrt{\kappa_{s} H_{a}},
$$


where $h_{f}$ is the height of the screen above the substrate, while $\kappa_{s}$ and $H_{a}$ are the curvature and height of the squeegee tip. Significant improvements in the process may be achieved by varying these geometric parameters; for example, (61) shows that the flux of paste through the screen near the tip is directly proportional to $\delta_{0}$.

An interesting generalisation of our model occurs if $D$ is sufficiently small to be comparable with $\left(\varepsilon \tau_{0}\right)^{2}$, in which case the limits $\varepsilon \rightarrow 0$ and $D \rightarrow 0$ do not commute. Our asymptotic analysis of the peeling region in Section 6 then ceases to be valid, and (69a) must be modified to

$$
g_{t}=g_{\zeta \zeta}-\left(\frac{\varepsilon \tau_{0}}{12 \sqrt{D}}\right)\left(g^{3} g_{\zeta \zeta \zeta}\right)_{\zeta}
$$

This extends McEwan and Taylor's model [24] for the peeling of a flexible strip attached by a viscous adhesive, by including permeability of the strip. This limit also has similarities with models of airway reopening $[25,26]$.

Our primary interest has been to investigate the behaviour near the squeegee tip. Our scalings in this inner region provide us with an aspect ratio and reduced Reynolds number that fully justify the use of Reynolds' equation. However, the validity of lubrication theory in the outer region is less certain; a future plan is to model this region using two-dimensional Stokes flow.

Our model can easily be applied to printing various patterns by allowing the permeability to be a function of position. Another obvious extension of our work would be to consider non-Newtonian paste. In addition, squeegees are often made of deformable rubber, and it would be relatively straightforward to incorporate squeegee deformation in our framework. In fact, we have shown that the pressure profile remains virtually constant in time, so that any squeegee deformation would likewise remain roughly constant throughout the print. The most serious deficiency in our model is the assumption that paste fills the entire domain. Our main priority is to model a finite volume of paste that initially sits above the screen before being forced through by the squeegee motion.

\section{Acknowledgements}

The authors are grateful to EPSRC and Du Pont (UK) Ltd. for their financial support. 


\section{References}

1. D.R. Kobs and D.R. Voigt, Parametric dependencies in thick film screening. Proc. ISHM 18 (1970) 1-10.

2. D.E. Riemer, Analytical model of the screen printing process: part 1. Solid State Technol. 8 (1988) 107-111.

3. D.E. Riemer, Analytical model of the screen printing process: part 2. Solid State Technol. 9 (1988) 85-90.

4. D.E. Riemer, The theoretical fundamentals of the screen printing process. Hybrid Circuits 18 (1989) 8-17.

5. G.I. Taylor, On scraping viscous fluid from a plane surface. Miszallangen Angewandten Mechanik (1962) 313-315.

6. B. Hunter, A Stokes flow analysis of the screen printing process. Int. J. Microcircuits and Electronic packaging 17 (1994) 21-26.

7. J. Riedler, Viscous flow in corner regions with a moving wall and leakage of fluid. Acta Mechanica 48 (1983) 95-102.

8. S.J. Chapman, A.D. Fitt and C.P. Please, Extrusion of power-law shear thinning fluids with small exponent. Int. J. Non-linear Mech. 32 (1997) 187-199.

9. J. Jeong and M. Kim, Slow viscous flow due to sliding of a semi-infinite plate over a plane. J. Phys. Soc. Jpn 54 (1985) 1789-1799.

10. J.A. Owczarek and F.L. Howland, A study of the off-contact screen printing process - part I: model of the printing process and some results derived from experiments. IEEE Trans. Comp. Hybrids Manuf. Technol. 13 (1990) 358-367.

11. J.A. Owczarek and F.L. Howland, A study of the off-contact screen printing process - part II: analysis of the model of the printing process. IEEE Trans. Comp. Hybrids Manuf. Technol. 13 (1990) 368-375.

12. J.T. Anderson, D.T. Gethin, T.C. Claypole, E.H. Jewell, M.F.J. Bohan and T.V. Korochkina, Hydrodynamic interactions in the screen printing process. J. Prepress and Printing Technol. 3 (2000) 155-192.

13. G.P. Glinski, C. Bailey and K.A. Pericleous, A non-Newtonian computational fluid dynamics study of the stencil printing process. Proc. Instn. Mech. Engrs. 215 (2001) 437-446.

14. H. Ockendon and J.R. Ockendon, Viscous Flow. Cambridge: Cambridge University Press (1995) 113pp.

15. K.K. Tio and S.S. Sadhal, Boundary conditions for stokes flow near a porous membrane. Applied Scientific Research 52 (1994) 1-20.

16. C. Pozrikidis, Shear flow over a particulate or fibrous plate. J. Eng. Math. 39 (2001) 3-24.

17. C. Pozrikidis, Boundary conditions for a shear flow past a permeable interface modeled as an array of cylinders. Computers and Fluids 33 (2004) 1-17.

18. C. Pozrikidis, Effect of membrane thickness on the slip and drift velocity in parallel shear flow. Journal of fluids and structures 20 (2005) 177-187.

19. A. Oron, S. H. Davis and S. G. Bankoff, Long-scale evolution of thin liquid films. Rev. Modern Phys. 69 (1997) 931-980.

20. I.S. Gradshteyn and I.M. Ryzhik, Table of Integrals, Series, and Products. Fifth Edition. San Diego: Academic Press (1994) 1204pp.

21. S.H. Davis and L.M. Hocking, Spreading and imbibition of viscous fluid on a porous base. Phys. Fluids 11 (1999) 48-57.

22. S.H. Davis and L.M. Hocking, Spreading and imbibition of viscous fluid on a porous base II. Phys. Fluids 12 (2000) 1646-1655. 
23. N. Alleborn and H. Raszillier, Spreading and sorption of a droplet on a porous substrate. Chem. Eng. Sci. 59 (2004) 2071-2088.

24. A.D. McEwan and G.I. Taylor, The peeling of a flexible strip attached by a viscous adhesive. J. Fluid Mech. 26 (1966) 1-15.

25. D.P. Gaver III, D. Halpern, O.E. Jensen and J.B. Grotberg, The steady motion of a semi-infinite bubble through a flexible-walled channel. J. Fluid Mech. 319 (1996) 25-65.

26. O.E. Jensen, M.K. Horsburgh, D. Halpern and D.P. Gaver III, The steady propagation of a bubble in a flexible-walled channel: asymptotic and computational models. Phys. Fluids 14 (2002) 443-457. 
ENGI_1509.tex; 10/06/2005; 14:10; p.32 